\title{
Experimental investigation of enhanced heat transfer of self-exciting mode oscillating-flow heat pipe with non-uniform profile under laser heating
}

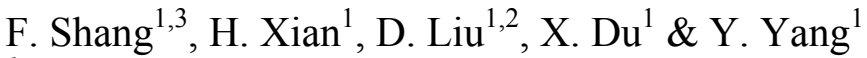 \\ ${ }^{I}$ North China Electric Power University, Beijing \\ People's Republic of China \\ ${ }^{2}$ Institute of Engineering Thermophysics, Chinese Academy of Sciences, \\ Beijing, People's Republic of China \\ ${ }^{3}$ Changchun Institute of Technology, Changchun \\ People's Republic of China
}

\begin{abstract}
The aim of this paper is to detail the heat transfer characteristics and transport power of the Self-Exciting Mode Oscillating Flow (SEMOS) Heat Pipe. The enhanced heat transfer performance of a non-uniform cross-section closed loop SEMOS HP is tested and compared with the uniform profile SEMOS HP under same heating conditions. As a result, the heat transfer rate of the SEMOS Heat Pipe with a non-uniform profile could be increased under certain conditions, which forms the basis for future research and development of new SEMOS Heat Pipes.

Keywords: laser heating, non-uniform profile SEMOS Heat Pipe, heat transfer enhancement, experimental investigation.
\end{abstract}

\section{Introduction}

A new era in heat pipes began with the Pulsating Heat Pipe, invented by H. Akachi in 1994, and later called the Self-Exciting Mode Oscillating-Flow Heat Pipe $[1,2]$ (SEMOS Heat Pipe). Although it has only been known about for ten years, the SEMOS Heat Pipe is attracting worldwide attention because of its 
immeasurable application potential. A lot of research about the SEMOS Heat Pipe has been done in Japan, U.S.A, Germany, Russia, Ukraine and China etc.

However, research is being done is to understand the working principles of the SEMOS Heat Pipe, as it is still in its initial stage. This new highly effective heat transfer device is attracting attention from all over the world in the field of heat transfer due to phase transition. Therefore the main challenge is to eliminate the contradiction between downsizing the cooling device and increasing the generated heat and to improve the heat-dissipating performance to a higher lever for all of the researchers.

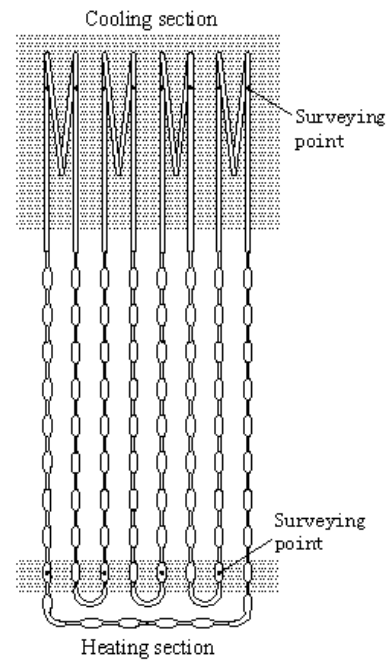

Figure 1: Experimental parts of a non-uniform profile of a SEMOS Heat Pipe.

\section{Heat transfer characteristics of the SEMOS Heat Pipe}

According to the working principles of the SEMOS Heat Pipe, there are two basic methods for improving the heat transfer performance: one is enhancing the heat transfer between the interior surface of the pipe and the working gas-liquid medium in the pipe either by increasing the phase-change frequency and intensity between liquid evaporation and gas clot or by improving the heat convection process between the working liquid and working surface because of the fact that the thermal conduction of the SEMOS Heat Pipe is between the pipe interface and two non-steady phases of working liquid in the pipe. The other method is increasing the pulsating frequency and reliable circulating power, which could be done by increasing the difference in temperature between the hot and cold fluid or by increasing the pulsing frequency inside the pipe. By enhancing the heat transfer rate of the SEMOS Heat Pipe, this experiment will 
validate the application of field cooperation theory on the heat transfer field with phase-change.

\section{Experiment setup}

The objects used in this experiment are SEMOS Heat Pipes with closed loops. One is a SEMOS Heat Pipe with a uniform profile of $3 \mathrm{~mm}$ in inner diameter, while the other heat pipe based on the uniform one with elliptic non-uniform profile consists of a vertically intervened heating section and insulating section of the pipe as shown in Figure 1. The pipe is made of brass, the working fluid in the pipe is distilled high-purity water with a filling rate $\varphi=42 \%$. The inclination angle of the heat pipe is $\theta=55^{\circ}$, and the pressure in the heat pipe is $P=1.8 \times 10^{-3} \mathrm{~Pa}$.

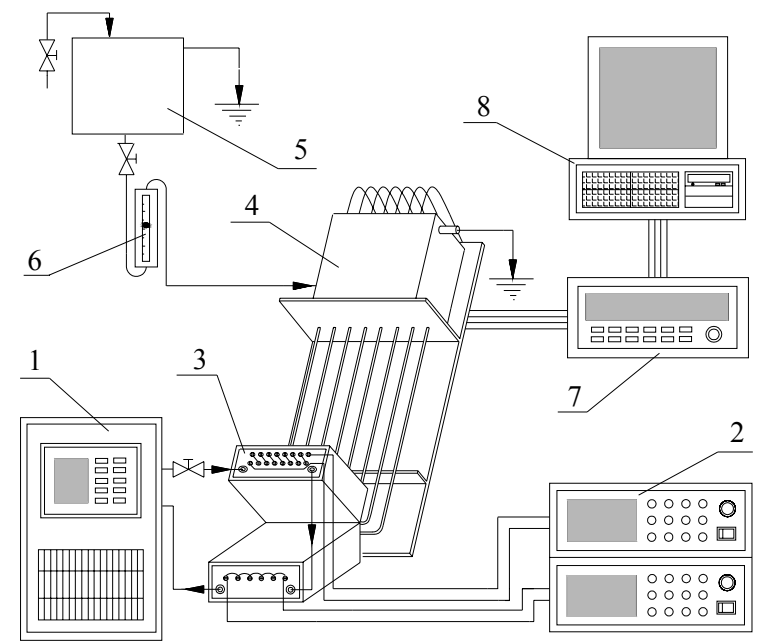

1-Unit of refrigeration cycle, 2-Power supply, 3-Laser supply, 4-Experimental table of SEMOS heat pipe, 5-Water tank, 6-Flow meter, 7-Data acquisition system, 8-Personal computer.

Figure 2: Experimental system of SEMOS Heat Pipe heat transfer enhancement.

Figure 2 shows the experimental setup for this thermal performance measurement. It consists of the main test apparatus: a laser supply and cooling system and the power supply system and a data acquisition system combined with a personal computer to show the data collected. As shown in Figure 2, the laser heater consists of 8-channel Quantum Well Laser Diode Arrays, while the maximum output power of every single channel is $50 \mathrm{~W}$. The heating electrical current range is 5 40A and the wave-length of the laser is $940 \mathrm{~nm}$. The heat input comes continuously from the eight laser heating channels, which could work individually or together as a heat source. A total of $20 \mathrm{~K}$-type thermocouples of 
$1 \mathrm{~mm}$ diameter were also added, which were mounted on the surface. Accordingly, the data acquisition frequency is $1 \mathrm{~Hz}$ and the data acquisition precision is at $0.01^{\circ} \mathrm{C}$.

\section{Results and discussion}

\subsection{Comparing and contrasting Heat Pipes with uniform and non-uniform profile}

Through the experiments mentioned above between the heat pipes with uniform profile and non-uniform profile, Figures 3, 4 and 5 show the change in efficiency in transport power under different heating powers (heat electrical current $I$ ). The efficiency transport power is given by the following equation:

$$
P_{o}=G c_{p}\left(T_{2}-T_{1}\right) \quad[\mathrm{W}]
$$

where $G$ denotes the cooling water $(\mathrm{kg} / \mathrm{s}) ; c_{p}$ is the specific heat of water under constant pressure; $\mathrm{J} /(\mathrm{kg} \cdot \mathrm{K})$ and $T_{2}$ and $T_{1}$ denote the input and output temperatures of cooling water.

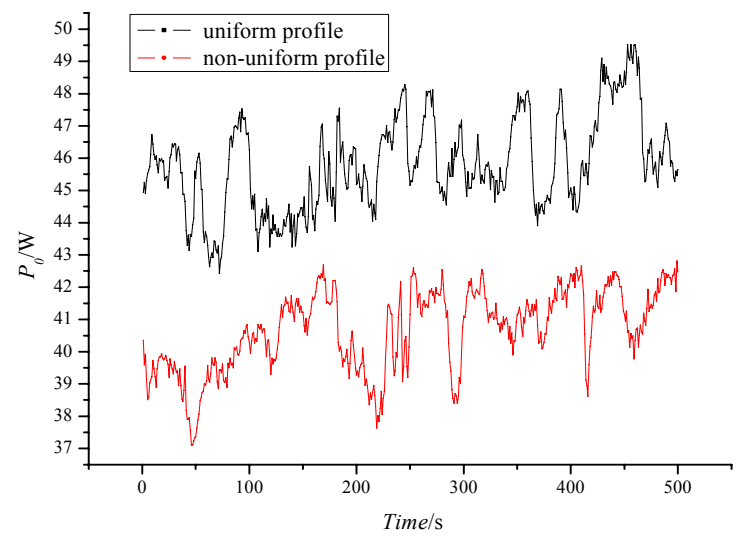

Figure 3: Comparison of transport power when heat electrical current is 14A.

As shown in Figures 3, 4 and 5, the transport power of the heat pipe with the non-uniform profile is lower than that of the heat pipe with the uniform profile when the heating electrical current is relatively low, and the rate of the heat pipe with the non-uniform profile would exceed that of the heat pipe with the uniform profile as the heating electrical current of the laser supply increases. It is demonstrated from the trend of the transfer rate $P_{o} \sim I$ graph that the greater the heat input, the greater the difference in transfer rate between the heat pipe with the non-uniform profile and the heat pipe with the uniform profile becomes. The transferred power of the heat pipe with the non-uniform profile is $13.6 \%$ higher 
than that of the heat pipe with the uniform profile at the maximum heat input (which, in this experiment is about $25.5 \mathrm{~W}$ and the heating electrical current of every channel is $23 \mathrm{~A}$.

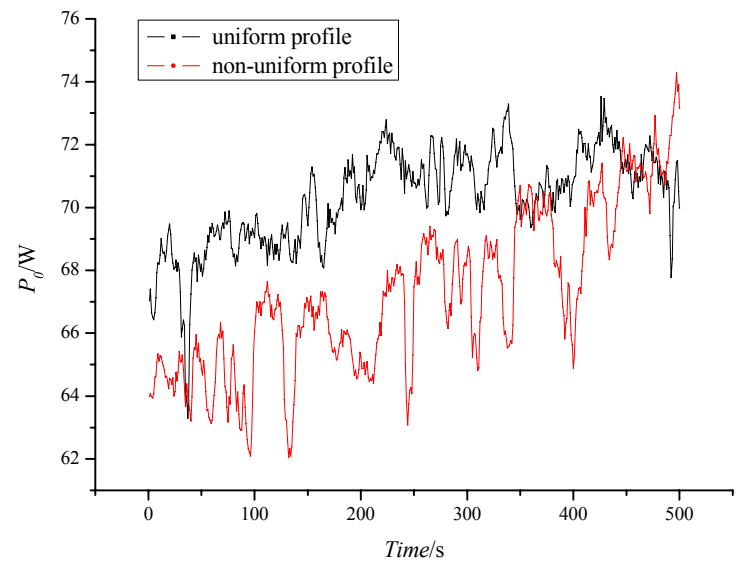

Figure 4: Comparison of transport power when heat electrical current is $18 \mathrm{~A}$.

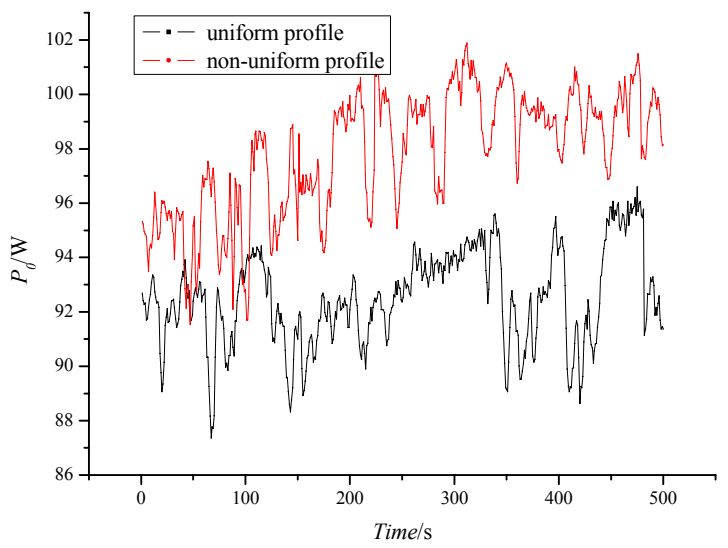

Figure 5: Comparison of transport power when heat electrical current is $23 \mathrm{~A}$.

\subsection{Comparison of effective thermal conductivity of uniform and non- uniform profiles and a pure conductor}

Figure 6 shows the relationship between the heat input and the effective thermal conductivity under three different conditions, SEMOS Heat Pipes with non-uniform profiles, with uniform profiles and the heat pipe without any working fluid which makes it a pure conductor. Under different outputs, and taking the difference in temperature between the hot and cold ends of the heat pipe, the effective thermal conductivity is shown below: 


$$
\lambda_{d}=\frac{P_{o} l}{\left(\overline{T_{h}}-\overline{T_{c}}\right) F} \quad[\mathrm{~W} /(\mathrm{m} \cdot \mathrm{K})]
$$

where $P_{o}$ denotes transport power; $\mathrm{W} ; l$ is the distance between the hot and cold end; $\mathrm{m} ; \overline{T_{h}}$ and $\overline{T_{c}}$ are the mean of the temperatures at the hot end and the cold end ${ }^{\circ} \mathrm{C} ; F$ is the total heat transfer area $\left(\mathrm{m}^{2}\right)$, which is the total circulate cross section area for heat transfer and the total cross section area for the pure conductor.

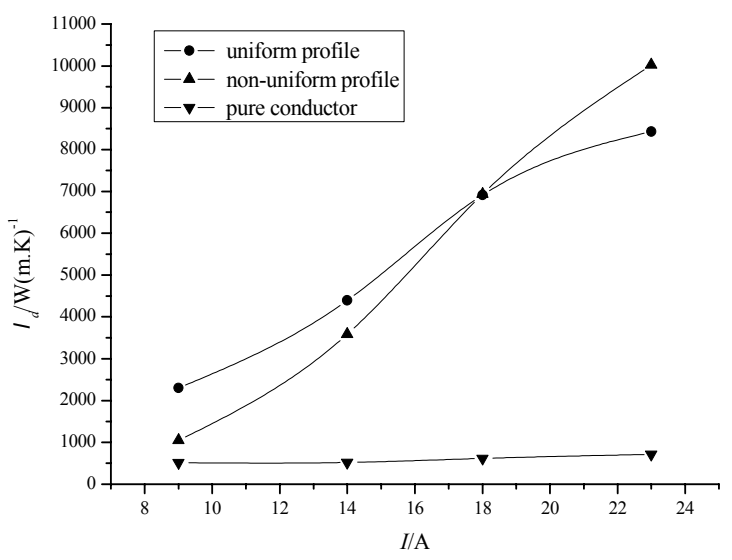

Figure 6: Comparison of effective thermal conductivity in varying cases.

As shown in Figure 6, the effective thermal conductivity increases with heat input in all three cases. The greatest increase in effective thermal conductivity is with the non-uniform heat pipe and the smallest increase is in the pure conductor heat pipe. When the heat input is low, the effective thermal conductivity of the uniform profile heat pipe is higher than that of the non-uniform one. As the heat input increases, the effective thermal conductivity of the non-uniform profile increases more than that of the uniform one and the difference between them becomes greater. It is suggested that under certain conditions, the heat transfer rate could be improved by changing the form of the profile of the SEMOS Heat Pipe. Figure 6 also shows that the function of heat transference of heat pipes especially SEMOS Heat Pipes is better than the performance of the heat pipe with the pure conductor. Within this heat input range, the effective thermal conductivity of the heat pipe is $4 \sim 15$ times that of the pure conductor, and this difference increases with heat input. The effective thermal conductivity of the non-uniform profile heat pipe is $19 \%$ higher than that of the one with the uniform profile and 14 times higher than that of the pure conductor at the maximum heat input (23A for every channel) in this experiment. 


\subsection{Heat transfer performance of the Heat Pipe with the non-uniform profile}

Theoretically, there are two reasons why a non-uniform heat pipe consisting of a heating section and an insulating section could improve the heat transfer rate. One is that the portrait eddy is formed on the particular section of the non-uniform profile, so that the vertical component of the velocity on the pipe surface increases, which means the temperature grads field and velocity vector field co-operate, which could improve the heat transfer rate. Meanwhile, the resistance on the working fluid increases because of the non-uniformity of the profile, so a certain amount of heat input is needed for the SEMOS Heat Pipe with the non-uniform profile to improve the heat transfer performance. The advantage of the SEMOS Heat Pipe with the non-uniform profile at improving heat transfer is easily demonstrated when the portrait eddy becomes stronger and circulating power declines, the SEMOS Heat Pipe with the non-uniform profile is more suitable for high density working fluid.

On the other hand, as shown in Figure 7, it is possible to conclude by monitoring the temperatures at the hot and cold end surface, that the oscillating amplitude of the non-uniform heat pipe of the surface temperature at the hot end is lower than that of the uniform one, while the oscillating frequency is higher. In the SEMOS Heat Pipe with the non-uniform profile, the circulating power increases because of the alternation of liquid evaporation and gas clot. The accelerated motion inside the pipe due to this unsteady expansion and contraction process along with the vertical velocity on the interface could enhance the heat transfer performance.

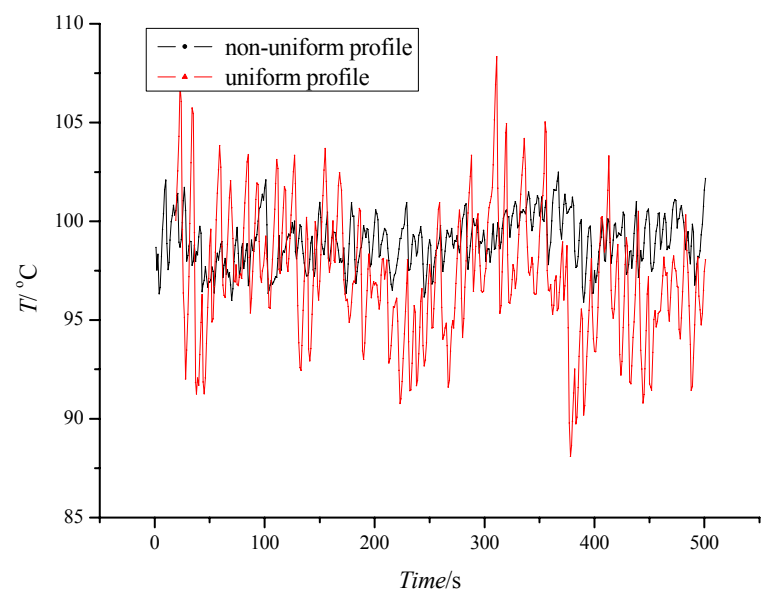

Figure 7: Comparison wave with the hot end surface temperature. 


\section{Conclusion}

a. The SEMOS Heat Pipe with the non-uniform profile can improve the heat transfer performance when the input heat is high enough to overcome the resistance brought on by the non-uniform profile.

b. Both the transmitted heat power of SEMOS Heat Pipes with non-uniform profile and uniform profile is much higher than that of the pure conductor under the same experimental conditions. The SEMOS Heat Pipe with the non-uniform profile is more suitable for high density heat current because of its particular advantage at high input levels.

c. The temperature oscillating frequency of the SEMOS heat pipe with the non-uniform profile becomes higher than that of the SEMOS Heat Pipe with the uniform profile as the heat input increases.

\section{References}

[1] H. Akachi, Looped capillary tube Heat Pipe. Proceedings of $71^{\text {st }}$ General Meeting Conference of JSME, pp. 940-950, 1994.

[2] H. Akachi, F. Polasek, P. Stulc, Pulsating Heat Pipe. Proceedings of the $5^{\text {th }}$ International Heat Pipe Symposium: Australia, pp.17-20, 1996.

[3] Qu Wei, Ma Tongze, Experimental investigation on flow and heat transfer of Pulsating Heat Pipe. Journal of Engineering Thermo physics, 23(5), pp. 596-598, 2002.

[4] Cui Xiaoyu, Weng Jianhua, M. Groll, Experimental investigation of heat performance for the copper/water Pulsating Heat Pipe. Journal of Engineering Thermo physics, 24(5), pp. 864-866, 2003.

[5] Meng Jian, Chen Zejing, Li Zhixin, and Guo Zengyuan, Field coordination analysis and convection heat transfer enhancement in Duct. Journal of Engineering Thermo physics, 24(4), pp. 652-654, 2003.

[6] Guo Zengyuan, Physical mechanism and control of heat convection: Speed and temperature Field Synergy. Chinese Science Bulletin, 45(19), pp. 2118-2122, 2000.

[7] Yasushi KATO, Takao NAGASAKI, and Yutaka ITO, Study on looped Heat Pipe with non-uniform cross section. Proceedings of the $40^{\text {th }}$ Heat Transfer Conference: Japan, pp. 313-314, 2003. 